\title{
The structure of atomic gas around the supernova remnant 3C 400.2
}

\author{
E.B. Giacani ${ }^{1}$, G. Dubner ${ }^{1}$, C. Cappa ${ }^{2}$, and J. Testori ${ }^{3}$ \\ 1 Instituto de Astronomía y Física del Espacio, CC 67, Suc. 28, 1428 - Buenos Aires, Argentina \\ e-mail: egiacani@iafe.uba.ar, gdubner@iafe.uba.ar \\ 2 Facultad de Ciencias Astronómicas y Geofísicas, UNLP, Argentina \\ e-mail: ccappa@fcaglp.fcaglp.unlp.edu.ar \\ 3 Instituto Argentino de Radioastronomía, Argentina \\ e-mail: jtestori@irma.edu.ar
}

Received April 7; accepted May 14, 1998

\begin{abstract}
The interaction of the SNR 3C 400.2 with the interstellar medium is investigated on the basis of the HI observations using the Synthesis Radio Telescope at the Dominion Radio Astrophysical Observatory (DRAO). These new data show the existence of a dense atomic cloud associated with $3 \mathrm{C} 400.2$ in the velocity range +14 to $+42 \mathrm{~km} \mathrm{~s}^{-1}$, and high velocity clouds probably accelerated by the supernova blast wave at $v=-49.5 \mathrm{~km} \mathrm{~s}^{-1}$ and $+69 \mathrm{~km} \mathrm{~s}^{-1}$. We propose that the complex remnant $3 \mathrm{C} 400.2$ is the result of a supernova explosion in a region of the interstellar medium containing a density discontinuity. A breakout phenomenon may explain the observed morphology in the different spectral regimes.
\end{abstract}

Key words: supernova remnants (SNRs) - supernova remnants: individual (3C 400.2) — radio lines: ISM ISM: structure

\section{Introduction}

3C 400.2 (G 53.6 - 2.2) belongs to a poorly understood class of supernova remnants (SNRs) characterized by a non-thermal radio shell, and thermal $\mathrm{X}$-ray emission filling the interior.

Based on VLA observations performed in the radiocontinuum at 327.5 and $1465 \mathrm{MHz}$, Dubner et al. (1994) have shown that $3 \mathrm{C} 400.2$ has a complex morphology which resembles two synchrotron shells that overlap on the northwestern side of the remnant. The larger shell in the southeast, looks almost complete and is centered near J2000:

Send offprint requests to: E. Giacani

* E.B.G., G.D. and C.C., are Members of the Carrera del Investigador Científico, CONICET, Argentina.

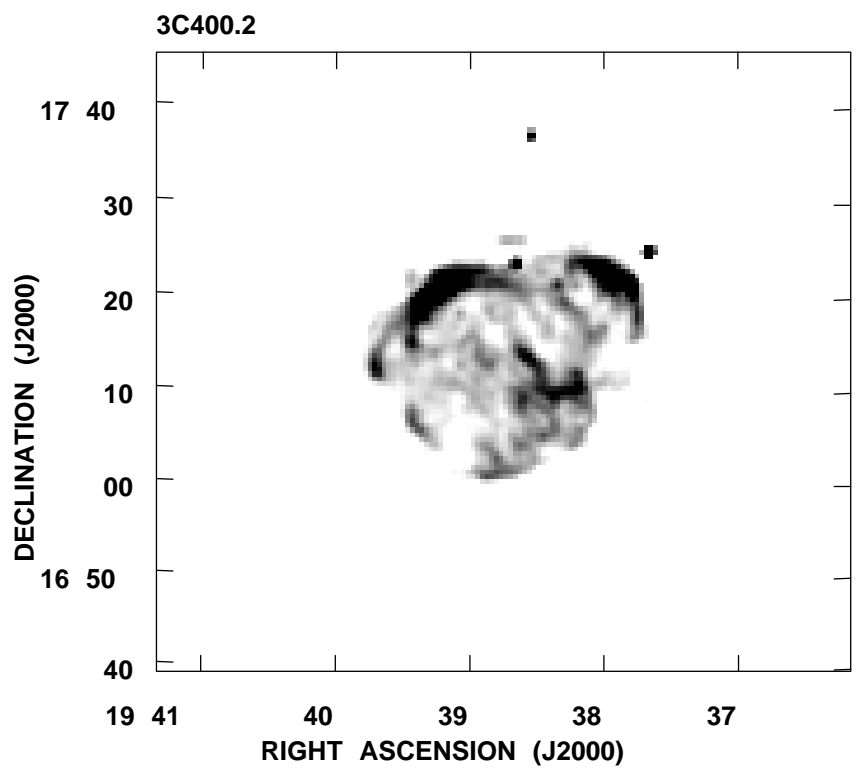

Fig. 1. Grayscale of the SNR 3C 400.2 at $1465 \mathrm{MHz}$ as taken from Dubner et al. (1994)

$19^{\mathrm{h}} 38^{\mathrm{m}} 53^{\mathrm{s}}, 17^{\circ} 12^{\prime} 55^{\prime \prime}$, the smaller one towards the northwest, is less complete and centered near J2000: $19^{\mathrm{h}} 38^{\mathrm{m}} 10^{\mathrm{s}}$, $17^{\circ} 17^{\prime} 52^{\prime \prime}$ (Fig. 1). In the present paper we will refer to them as the "large shell" and the "small NW shell", respectively. The interior $\mathrm{X}$-ray emission is thermal in nature and roughly ellipsoidal in shape. The brightest $\mathrm{X}$-ray peak is attained in the region of superposition of the two radio rings, over the edge of the larger circle and close to the center of the smaller one (Seward 1990; Saken et al. 1995). The X-ray data have been interpreted in terms of White \& Long's (1991) model of evaporation of embedded cloudlets. In the optical range, Winkler et al. (1993) obtained in the $\mathrm{H} \alpha$ and $\left[\mathrm{S}_{\mathrm{II}}\right]$ lines the only images that cover 
the full extent of $3 \mathrm{C} 400.2$. The optical filaments appear clearly correlated with the portions of the radio rings that overlap in the NW (Dubner et al. 1994).

It is important to discern the influence of the characteristics of the surrounding interstellar medium on the morphology of this SNR in the different spectral regimes. In that sense, the investigation of the distribution and kinematics of the neutral hydrogen around the SNR is a very useful tool, because it can provide a threedimensional picture of the environs where the remnant evolves and may help to distinguish among alternative scenarios that can result in similar morphologies.

In order to investigate the environs of $3 \mathrm{C} 400.2$ we carried out HI $\lambda 21 \mathrm{~cm}$ line observations in a field of $2.1 \times 2.1$ centered on J2000: $19^{\mathrm{h}} 38^{\mathrm{m}},+17^{\circ} 13^{\prime}$, using the DRAO Synthesis Telescope. Based on these observations we find that a possible scenario which simultaneously represents the $\mathrm{X}$-ray, optical, radio continuum and $\mathrm{HI}$ characteristics of $3 \mathrm{C} 400.2$, is a supernova exploding near the border of a clumpy neutral cloud about 5 times denser than its surroundings.

\section{Observations}

3C 400.2 was observed in the $\lambda 21$-cm HI line using the Synthesis Telescope at the Dominion Radio Astrophysical Observatory (DRAO) (Roger et al. 1973; Veidt et al. 1985) in June 1995. The field of view is $2.1 \times 2.1$ and the synthesized beam at the declination of $3 \mathrm{C} 400.2$ is $1.2 \times 1^{\prime}$.

Spectral information on the HI 21-cm line was detected in a 128 channels digital spectrometer, which were spaced by $1.65 \mathrm{~km} \mathrm{~s}^{-1}$, covering the velocity range from $-103.9 \mathrm{~km} \mathrm{~s}^{-1}$ to $+105.6 \mathrm{~km} \mathrm{~s}^{-1}$ (all velocities are relative to the Local Standard of Rest).

The observed visibilities were calibrated and transformed into sky images using the standard software of DRAO. Radio continuum emission at $1420 \mathrm{MHz}$ was subtracted from each line channel, and afterwards the HI line maps were corrected for the polar diagram of the antennas using a gaussian function of 105 arcmin FWHM. The data corresponding to shorter spacings were obtained from single dish $\mathrm{HI}$ observations carried out with the DRAO $26 \mathrm{~m}$ telescope using identical radial velocity and bandwidth parameters.

For presentation purposes, a constant background equal to the mean value of each channel map was subtracted from every map. Figure 2 shows the subtracted spectrum.

\section{Results}

\subsection{HI in the environs of $3 C 400.2$}

Figure 2 serves to illustrate the general HI emission in a region around $3 \mathrm{C} 400.2$. Significant HI emission is detected

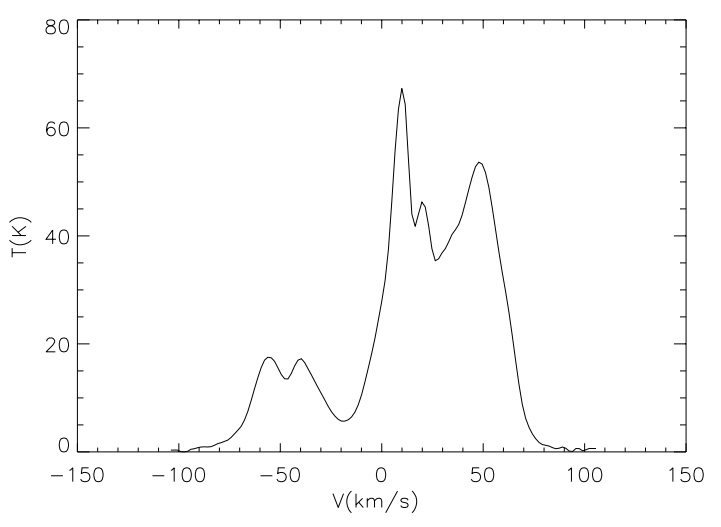

Fig. 2. Average HI spectrum substracted from the channel maps of HI emission

in the velocity interval $\simeq(-70,+70) \mathrm{km} \mathrm{s}^{-1}$. The emission which dominates the spectrum in the range $-10 \mathrm{~km} \mathrm{~s}^{-1}$ to $+15 \mathrm{~km} \mathrm{~s}^{-1}$ is usually associated with the local spiral arm; while emission at higher velocities can be related to the Carina-Sagittarius spiral arm, which is seen longitudinally in this direction of the Galaxy (Georgelin \& Georgelin 1976). According to circular galactic rotation models (e.g. Brand \& Blitz 1993), positive velocities higher than $\simeq+45 \mathrm{~km} \mathrm{~s}^{-1}$ are forbidden; however, as it is clear from this figure, strong HI emission is still detected up to $\simeq+70 \mathrm{~km} \mathrm{~s}^{-1}$. Emission at negative velocities should correspond to very distant gas, beyond the Solar Circle $(d \geq$ $10 \mathrm{kpc})$.

We examined in detail the HI 21-cm line data in the whole observed velocity interval looking for traces of expanding events like holes, shell-like features, etc. The HI data can also give information about the existence of neighbouring neutral clouds that may have been collided by a strong SN shock or are part of the population of cloudlets that are currently being evaporated, resulting in the observed thermal X-ray emission.

Figure 3 displays the $\mathrm{HI}$ emission distribution in the velocity interval $-55 \mathrm{~km} \mathrm{~s}^{-1}$ to $+74 \mathrm{~km} \mathrm{~s}^{-1}$. Each HI line map was obtained by averaging 3 individual maps, producing images with a velocity resolution of $\sim 5.0 \mathrm{~km} \mathrm{~s}^{-1}$, and convolved to a beam size of 3 arcmin. Superimposed on the HI images are the 2, 6 and $10 \mathrm{mJy} /$ beam contours of the radio continuum emission of $3 \mathrm{C} 400.2$ at $1465 \mathrm{MHz}$ as taken from Dubner et al. (1994). The central velocity of each map is indicated in the upper left corner. The same grayscale is used for the whole velocity interval to help a quick visual identification of maxima and minima in the emission along the line of sight.

In the images at -49.5 and $-44.5 \mathrm{~km} \mathrm{~s}^{-1}$ a small patch of emission is seen projected onto the center of the remnant. Based on the positional coincidence with the remnant and on the fact that this feature is unique, we 


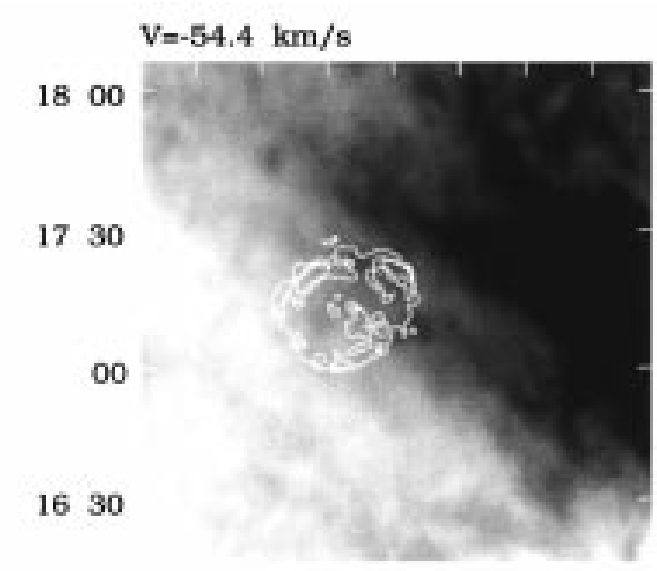

$\begin{array}{llllllll}19 & 41 & 40 & 39 & 38 & 37 & 36 & 35\end{array}$

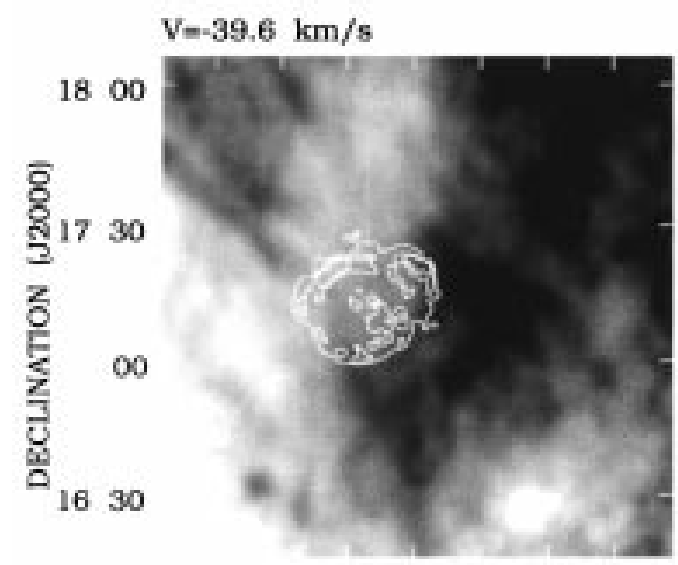

$\begin{array}{llllllll}19 & 41 & 40 & 39 & 38 & 37 & 36 & 35\end{array}$

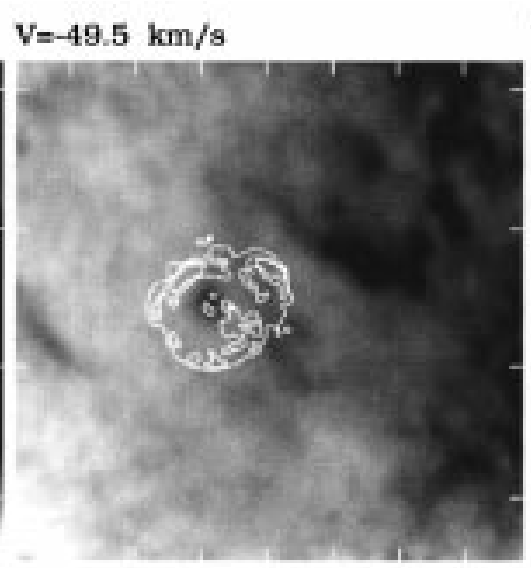

$\begin{array}{lllllll}41 & 40 & 39 & 38 & 37 & 36 & 35\end{array}$

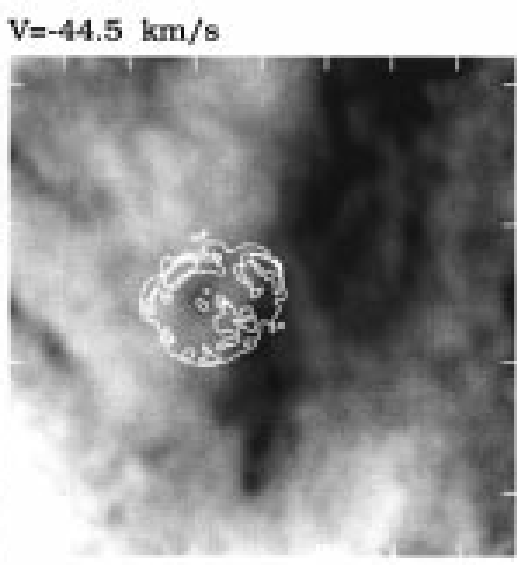

$\begin{array}{lllllll}41 & 40 & 39 & 38 & 37 & 36 & 35\end{array}$

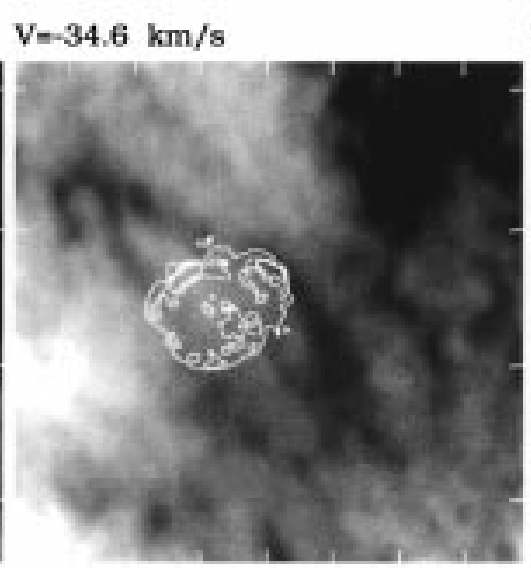

$\begin{array}{lllllll}41 & 40 & 39 & 38 & 37 & 36 & 35\end{array}$

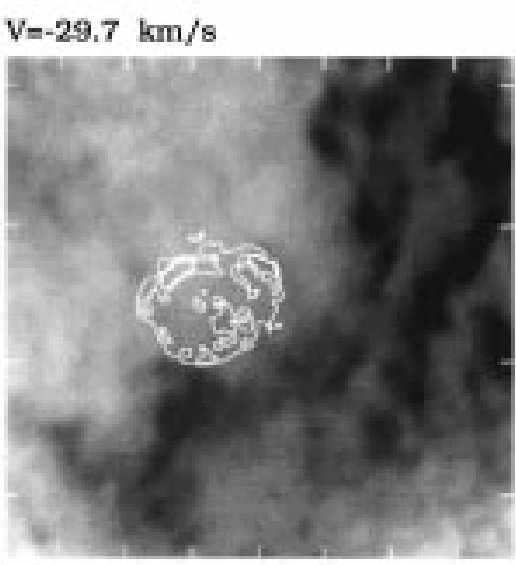

$\begin{array}{lllllll}41 & 40 & 39 & 38 & 37 & 36 & 35\end{array}$

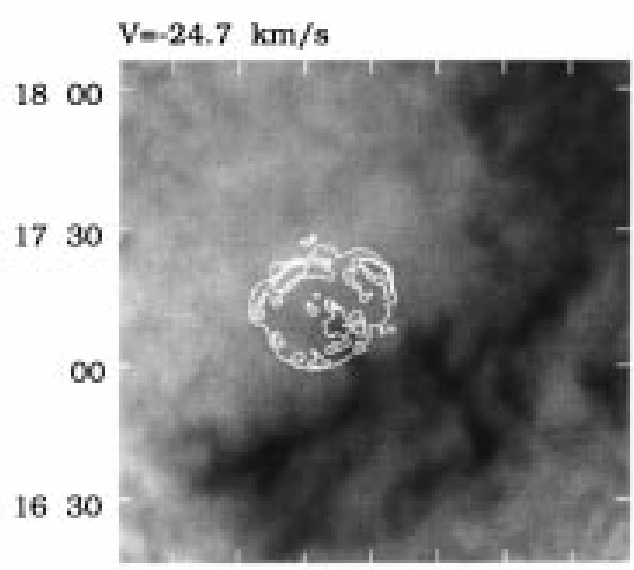

$\begin{array}{llllllll}19 & 41 & 40 & 39 & 38 & 37 & 36 & 35\end{array}$

\section{$\mathrm{V}=-19.8 \mathrm{~km} / \mathrm{s}$}

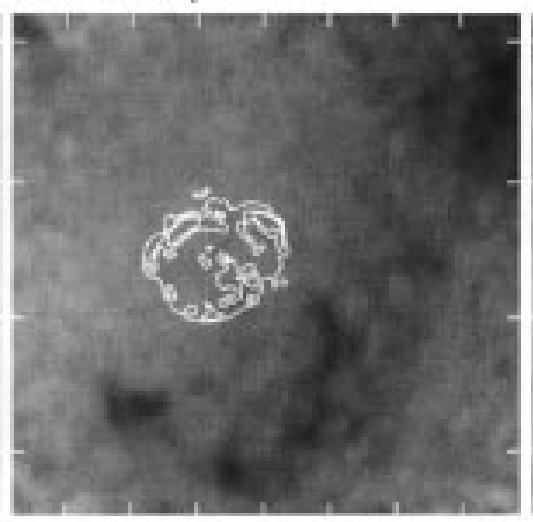

$\begin{array}{lllllll}41 & 40 & 39 & 38 & 37 & 36 & 35 \\ \text { RIGHT } & \text { ASCENSION } & (\text { J200O) }\end{array}$
$\mathrm{V}=-14.8 \mathrm{~km} / \mathrm{s}$

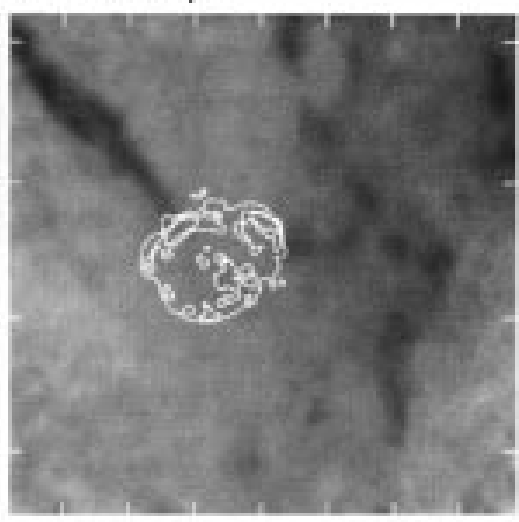

$\begin{array}{lllllll}41 & 40 & 39 & 38 & 37 & 36 & 35\end{array}$

Fig. 3. Grayscale images of 3C 400.2 in the neutral hydrogen $\lambda 21 \mathrm{~cm}$ line in the velocity range $v=-54.4$ to $+74.2 \mathrm{~km} \mathrm{~s}$. The grayscale goes linearly from -10 to $+15 \mathrm{~K}$. Each image is an average of three channels. The central velocity is indicated in the upper left corner. Superimposed on the HI images are the 2, 6 and $10 \mathrm{mJy} /$ beam contours of the radio continuum emission of 3C 400.2 at $1465 \mathrm{MHz}$ as taken from Dubner et al. (1994) 

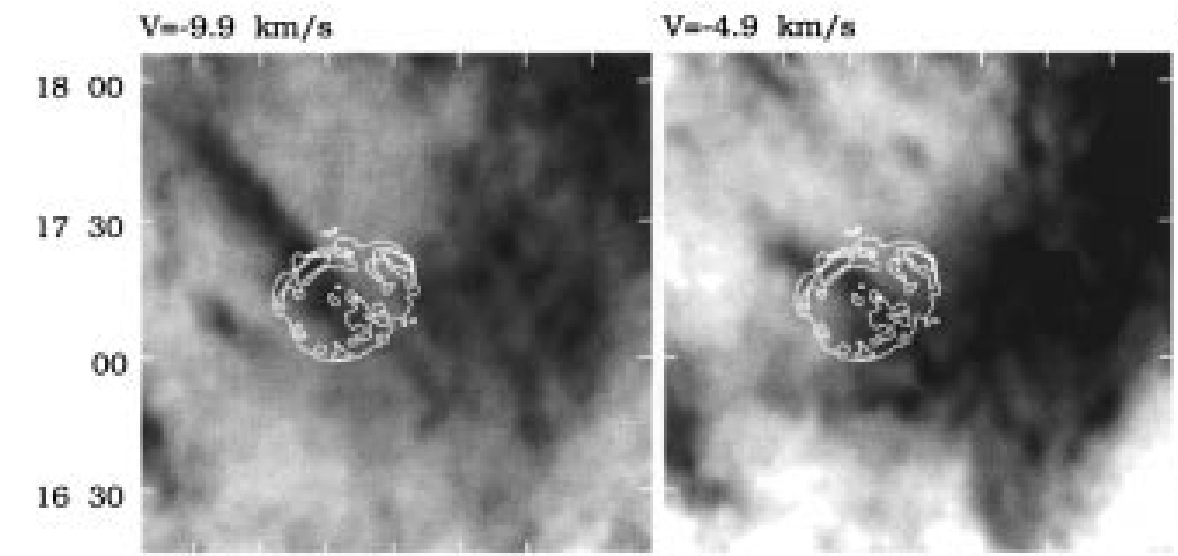

$\begin{array}{llllllll}19 & 41 & 40 & 39 & 38 & 37 & 36 & 35\end{array}$ $\begin{array}{lllllll}41 & 40 & 39 & 38 & 37 & 36 & 35\end{array}$

\section{$\mathrm{V}=+9.9 \mathrm{~km} / \mathrm{s}$}

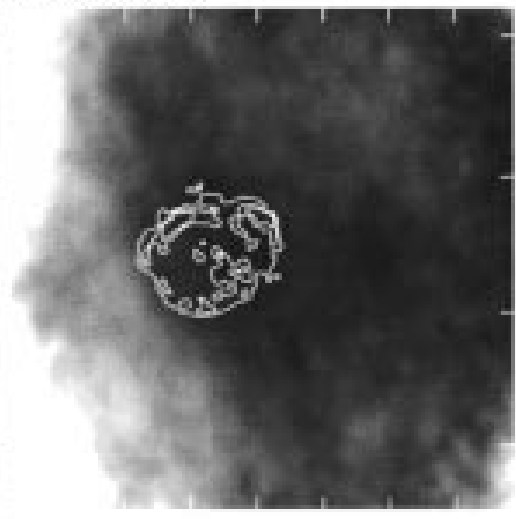

$\begin{array}{lllllll}41 & 40 & 39 & 38 & 37 & 36 & 35\end{array}$
$\mathrm{V}=0.0 \mathrm{~km} / \mathrm{s}$

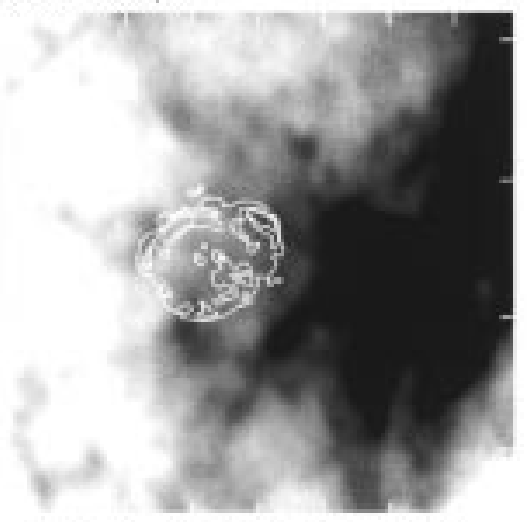

$\begin{array}{lllllll}41 & 40 & 39 & 38 & 37 & 36 & 35\end{array}$

$\mathrm{V}=+14.8 \mathrm{~km} / \mathrm{s}$

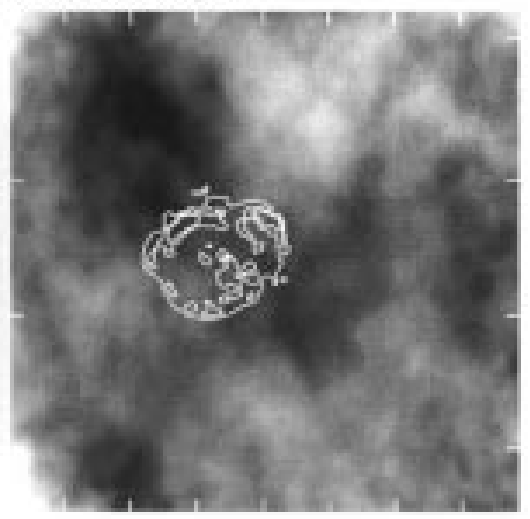

$\begin{array}{lllllll}41 & 40 & 39 & 38 & 37 & 36 & 35\end{array}$

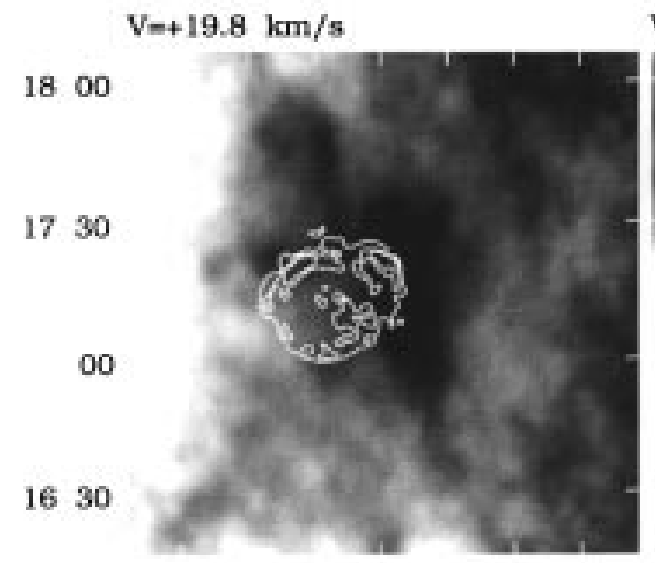

$\begin{array}{llllllll}19 & 41 & 40 & 39 & 38 & 37 & 36 & 35\end{array}$
$\mathrm{V}=+24.7 \mathrm{~km} / \mathrm{s}$

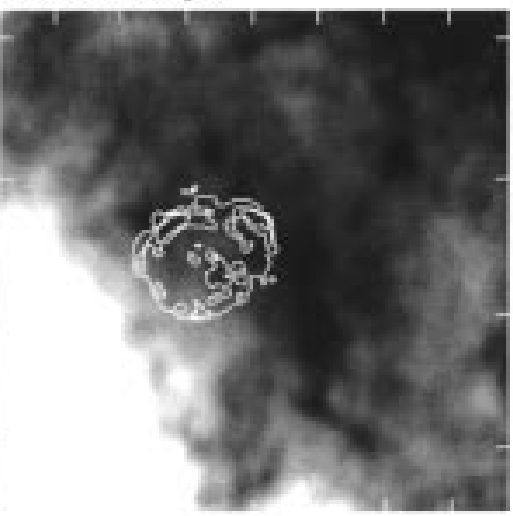

$\begin{array}{lllllll}41 & 40 & 39 & 38 & 37 & 36 & 35\end{array}$ RIGHT ASCENSION (J2000)
$\mathrm{V}=+29.7 \mathrm{~km} / \mathrm{s}$

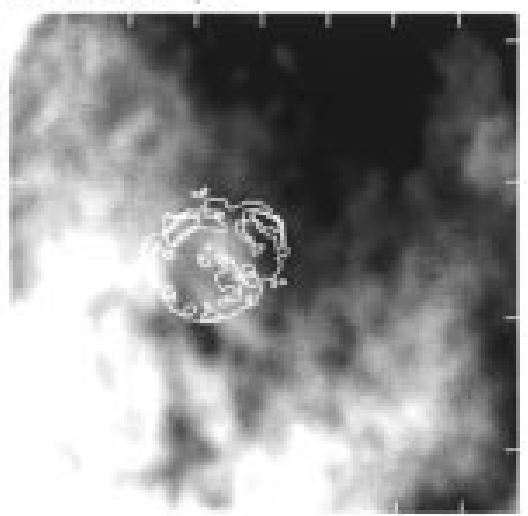

$\begin{array}{lllllll}41 & 40 & 39 & 38 & 37 & 36 & 35\end{array}$

Fig. 3. continued 

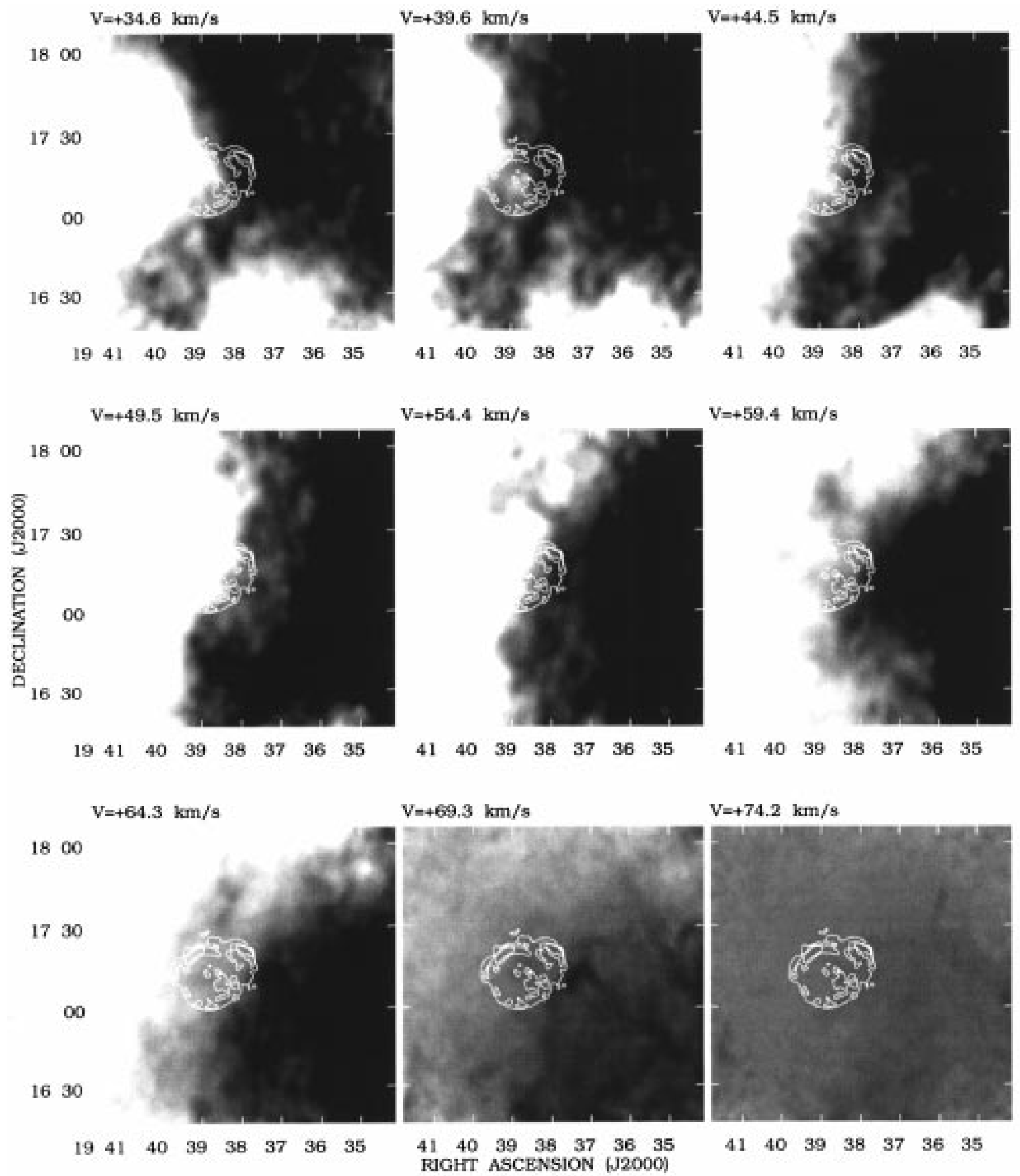

Fig. 3. continued 


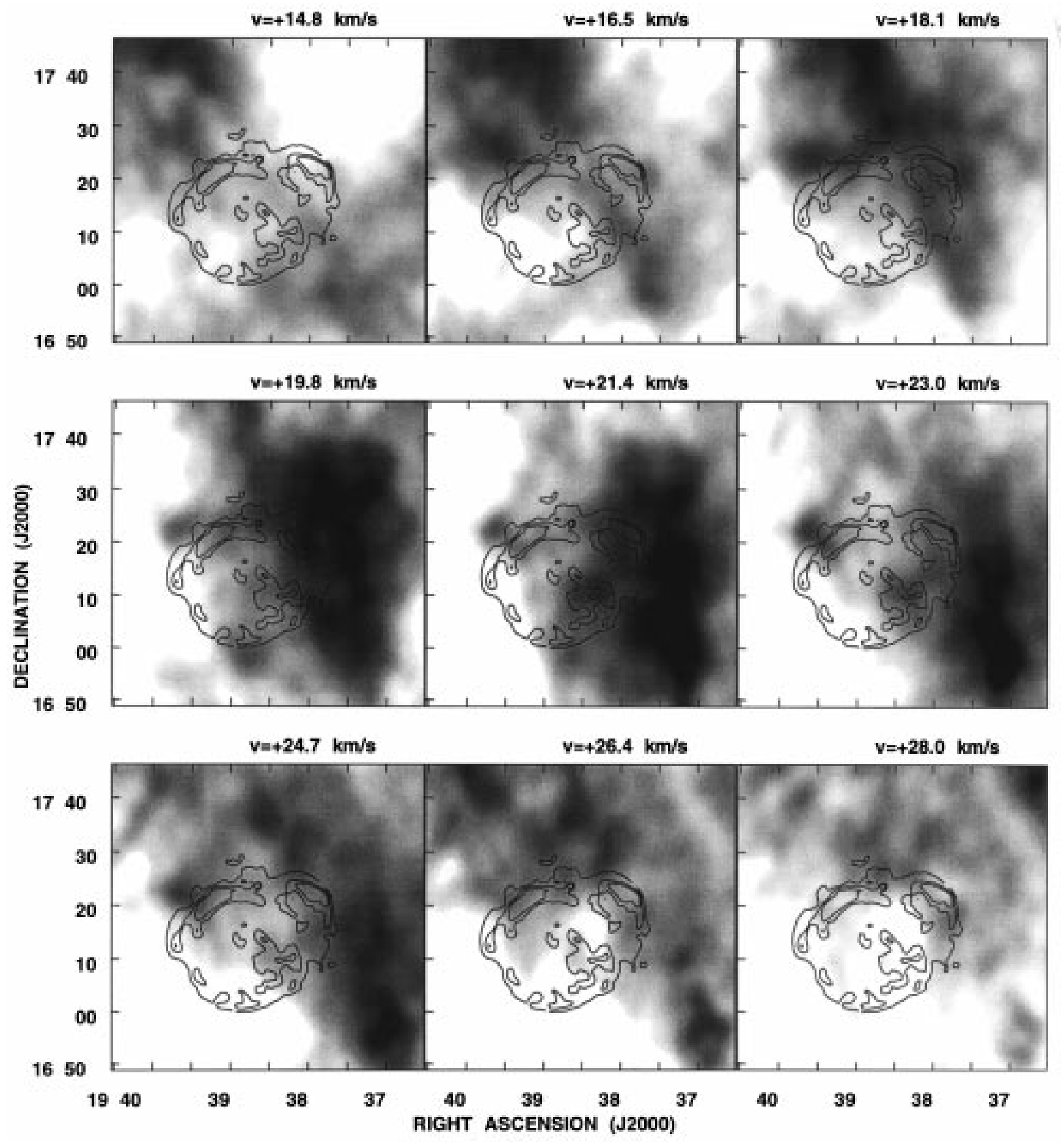

Fig. 4. Grayscale images of $3 \mathrm{C} 400.2$ in the neutral hydrogen $\lambda 21 \mathrm{~cm}$ line in the velocity range $v=+14.8$ to $+41.2 \mathrm{~km} \mathrm{~s}$ each $1.65 \mathrm{~km} \mathrm{~s}^{-1}$. The grayscale goes linearly from 0 to $20 \mathrm{~K}$. The central velocity is indicated in the upper right corner. The $1465 \mathrm{MHz}$ continuum image has been included on every image 


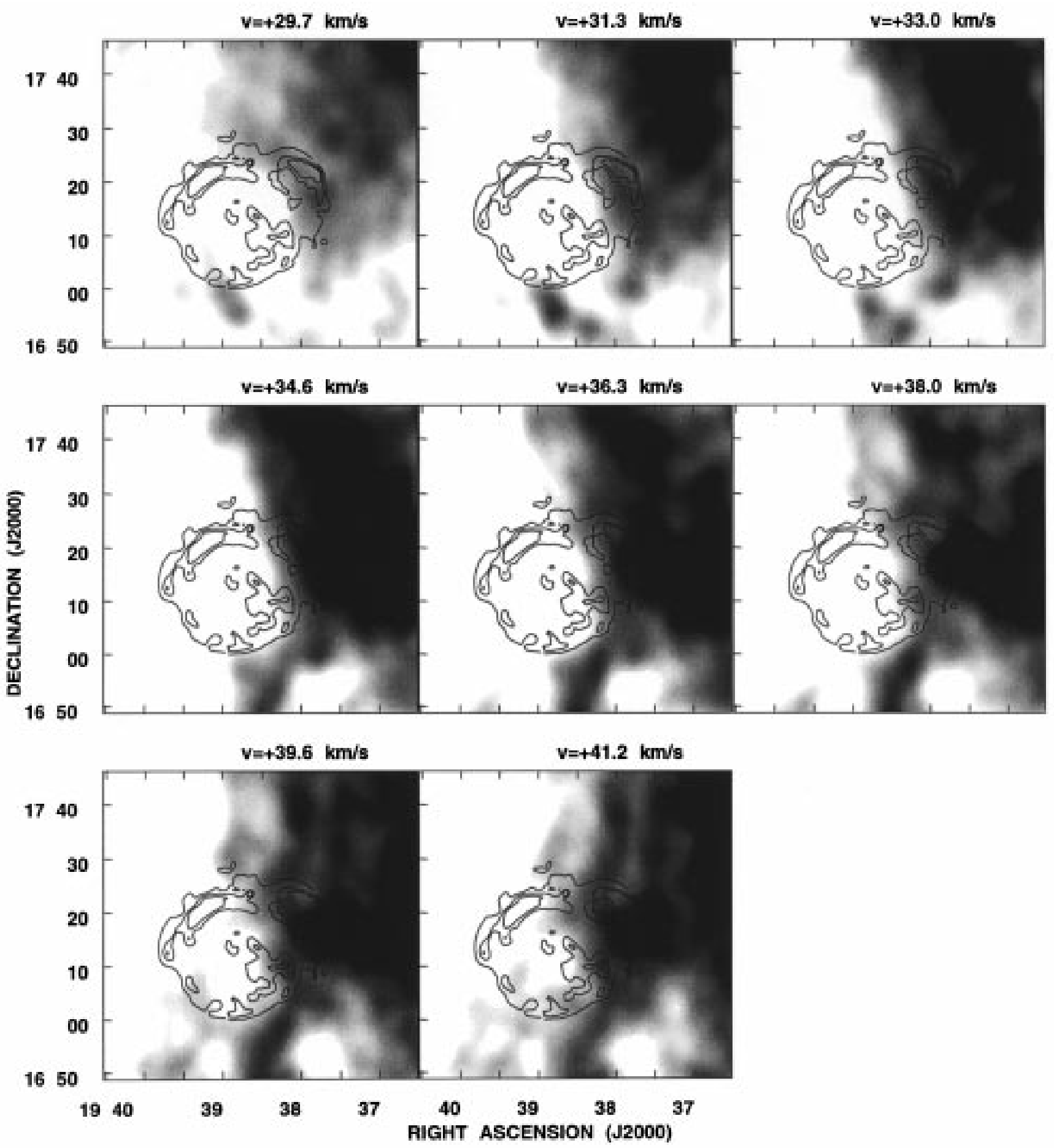

Fig. 4. continued

propose that it is a cloud that may have been accelerated by the $\mathrm{SN}$ shock.

At $v=-39.6$ and $-34.6 \mathrm{~km} \mathrm{~s}^{-1}$ the emission is patchy; no particular structure is likely to be associated with the SNR. From $v=-29.7$ to $-19.7 \mathrm{~km} \mathrm{~s}^{-1}$ the HI emission distribution appears almost perpendicular to the galactic plane orientation. Again its relation to the remnant is not evident. Between -14.8 and $-4.9 \mathrm{~km} \mathrm{~s}^{-1}$ a thin filament of neutral gas can be seen running through 3C 400.2 in a direction almost parallel to the galactic plane.

At positive velocities, the galactic background contribution is important, and the HI structures that are candidates for coupling with the radio continuum emission of 3C 400.2 need to be disentangled from the field emission. From 0 to $+9.9 \mathrm{~km} \mathrm{~s}^{-1}$ clumpy emission is detected 
in the whole observed field. In the velocity range +14.8 to $+29.7 \mathrm{~km} \mathrm{~s}^{-1}$ the most outstanding feature is the extended HI cloud, brighter at $+19.8 \mathrm{~km} \mathrm{~s}^{-1}$, which overlaps the west and north portions of $3 \mathrm{C} 400.2$. Part of this cloud appears also delineating the northeast radio maximum of the remnant centered around $19^{\mathrm{h}} 39^{\mathrm{m}} 30^{\mathrm{s}},+17^{\circ} 20^{\prime}$ (see Fig. 1).

From +34.6 to $+44.5 \mathrm{~km} \mathrm{~s}^{-1}$ the HI emission forms an incomplete ring-shaped feature surrounding the southern and western border of the "large shell".

For velocities greater than $v \simeq+50 \mathrm{~km} \mathrm{~s}^{-1}$ the only neutral features likely to be related to the remnant are a very weak cloudlet seen projected onto the center of the SNR at $v=+69.3 \mathrm{~km} \mathrm{~s}^{-1}$, and a small flattened cloud that appears "touching" the western border of 3C 400.2 at +64.3 and $+69.3 \mathrm{~km} \mathrm{~s}^{-1}$.

In our search for HI features associated with 3C 400.2, the structures seen between +15 and $+42 \mathrm{~km} \mathrm{~s}^{-1}$ appear particularly promising, since they show several morphological correspondences with the radio continuum emission. Figure 4 gives a more detailed view of the neutral gas distribution in the local neighbourhood of 3C 400.2 in the mentioned velocity range. The HI emission is displayed with a velocity resolution of $1.65 \mathrm{~km} \mathrm{~s}^{-1}$ using the same grayscale for the whole set of images. Again the 2,6 and $10 \mathrm{mJy} /$ beam contours of the radio continuum emission are superimposed.

From this figure, it can be observed that the overall HI distribution embraces most of the periphery of $3 \mathrm{C} 400.2$, opening in the direction opposite to the galactic plane.

Comparison between HI emission distribution and radio continuum emission show several good correspondences:

(1) from $v=+16.5$ to $+28 \mathrm{~km} \mathrm{~s}^{-1}$, the HI closely surrounds the NE, $\mathrm{N}$ and $\mathrm{W}$ borders of $3 \mathrm{C} 400.2$. In order to remark all the interesting matchings, in Fig. 5a we display the $\mathrm{HI}$ integrated between +18 and $+21 \mathrm{~km} \mathrm{~s}^{-1}$ in contourlines with $3 \mathrm{C} 400.2$ in grayscale. A perfect fitting between radio features and surrounding gas is observed towards both, the NE and NW radio continuum maxima;

(2) at $v=+26.4$ and $+28 \mathrm{~km} \mathrm{~s}^{-1}$ a hole in the HI distribution is noticeable centered near $19^{\mathrm{h}} 38^{\mathrm{m}} 26^{\mathrm{s}},+17^{\circ} 15^{\prime}$. This minimum coincides with the interior of the "small NW shell" of 3C 400.2. Moreover, the SE wall of the HI cavity exactly overlaps the SE border of that radio shell (see particularly the image at $v=+26.4 \mathrm{~km} \mathrm{~s}^{-1}$ ). This suggested association between the HI features and the remnant, is reinforced by the fact that the HI features also match the optical and the X-ray emission observed to be associated with $3 \mathrm{C} 400.2$. Figure $5 \mathrm{~b}$ displays the $\mathrm{H}_{\alpha}$ emission (greyscale) as taken from Winkler et al. (1993) with a few HI contourlines overlapped. It can be appreciated that most of the $\mathrm{H}_{\alpha}$ filaments correspond with regions of higher compression in the HI contours, and the center of the HI void coincides with the geometrical center of the
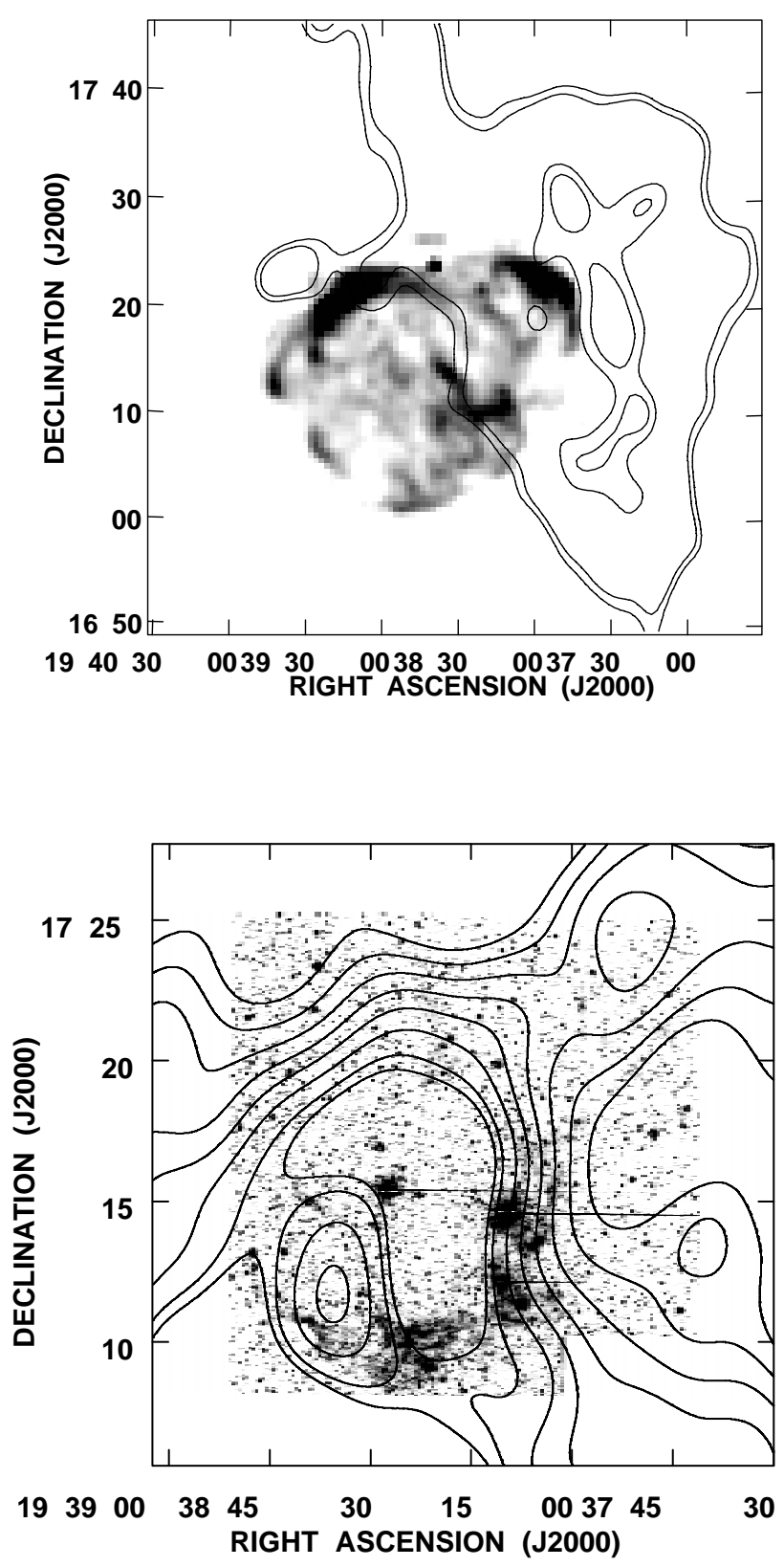

Fig. 5. a) Overlay of the 1465 radio continuum image of 3C 400.2 as obtained by Dubner et al. (1994), with some contours of the HI distribution integrated between +18 and $+21 \mathrm{~km} \mathrm{~s}^{-1}$. b) Overlay of the $\mathrm{H} \alpha+[\mathrm{NII}]$ CCD image of 3C 400.2, as obtained by Winkler et al. (1993), with some contours of the HI distribution at $v=+26.4 \mathrm{~km} \mathrm{~s}^{-1}$

optical shell. Also, from the visual comparison between the X-ray image of 3C 400.2 (see for example, Fig. 2 in Saken et al. 1995) and the HI emission, particularly at $v=+28 \mathrm{~km} \mathrm{~s}^{-1}$, it is found that the HI closely surrounds the X-ray emission along the eastern side; and

(3) from $v \simeq+31$ to $+42 \mathrm{~km} \mathrm{~s}^{-1}$ the most interesting feature is the band of neutral gas that exactly embraces the west and south rim of the "large shell". This excellent correspondence allows one to conclude that they are in physical contact. Note that in this last velocity range the 
"small NW shell" is increasingly covered by the HI cloud and only the "large shell" appears interacting with the HI.

Based on the present HI results, we propose that 3C 400.2 is the complex remnant resulting from a SN explosion occurring in the border of a dense HI cloud. In the proposed scenario the "small NW shell" is penetrating into the dense material, while the "large shell" is breaking out into a lower density medium. Thus, the expanding shock front pushed away neutral gas from the surroundings, transferring mechanical energy to the environs. We choose $v \simeq+27 \mathrm{~km} \mathrm{~s}^{-1}$ as the systemic velocity of the remnant based on the fact that the HI void is better seen at $v=+26.4$ and $+28 \mathrm{~km} \mathrm{~s}^{-1}$. Then, all the associated HI features seen between +14 and $+27 \mathrm{~km} \mathrm{~s}^{-1}$ should represent approaching gas. In this range we showed the existence of HI correlated with both the "small" and the "large" shells. The receding gas is seen at velocities between +27 and $+42 \mathrm{~km} \mathrm{~s}^{-1}$, and correlates only with the "large shell" (mainly towards the south).

\subsection{The distance to $3 C 400.2$}

Current estimates of the distance to 3C 400.2 based on the $\Sigma-\mathrm{D}$ relationship, range from 3.8 to $6.3 \mathrm{kpc}$ (Clark \& Caswell 1976; Caswell \& Lerche 1979; Milne 1979; Dubner et al. 1994); however this method is known to have large intrinsic dispersion. Rosado (1983) derived a distance of $6.7 \mathrm{kpc}$ from the kinematics of the optical filaments, but her estimate is highly uncertain because it is based on interferograms obtained in a small portion of the SNR. From our HI observations, adopting $+27 \mathrm{~km} \mathrm{~s}^{-1}$ as the systemic velocity of the SNR and using the galactic flat rotation curve by Fich et al. (1989), (with $R_{\odot}=8.5 \mathrm{Kpc}$ and $V_{\odot}=220 \mathrm{~km} \mathrm{~s}^{-1}$ ), we obtain the kinematical distances of $\simeq 2.3 \mathrm{kpc}$ and $\simeq 7.7 \mathrm{kpc}$. An uncertainty of $\simeq 0.8 \mathrm{kpc}$ can be estimated assuming non-circular motions of about $\pm 7 \mathrm{~km} \mathrm{~s}^{-1}$ (Burton 1992). Since the last value would correspond to an interarm region (Georgelin \& Georgelin 1976), we adopt the near kinematical distance as the most reliable for this SNR. At a distance of $2.3 \pm 0.8 \mathrm{kpc}$ the linear sizes of $3 \mathrm{C} 400.2$ are $15 \pm 5$ pc for the "large shell" and $9.4 \pm 3$ pc for the "small NW shell".

\subsection{Physical parameters}

According to the above scenario, the supernova explosion took place near $19^{\mathrm{h}} 38^{\mathrm{m}} \cdot 4,17^{\circ} 15^{\prime}$ and the shock front travelling towards the NW and W directions was slowed down by the presence of the HI cloud detected between +14 and $+42 \mathrm{~km} \mathrm{~s}^{-1}$ forming the $9.4 \mathrm{pc}$ "small NW shell". The "large shell" expanded into a lower density medium towards the SE.

To estimate the volumetric density of both the high and low density regions, we integrated the $\mathrm{HI}$ emission in the $(+14,+42) \mathrm{km} \mathrm{s}^{-1}$ velocity range and assumed that the gas is optically thin. In a three-dimensional picture we adopted for the HI dense cloud a depth at least equal to the diameter of the "large shell", since as it was shown above there are signs of physical interaction between the "large shell" and the external cloud at $v \simeq+40 \mathrm{~km} \mathrm{~s}^{-1}$. Using this model we obtained values of about $21 \mathrm{~cm}^{-3}$ and $4 \mathrm{~cm}^{-3}$ for the volume atomic densities for the dense cloud and the lower density medium respectively.

A total HI mass for the dense cloud of about $3000 M_{\odot}$ was calculated by adding all the emission associated to $3 \mathrm{C} 400.2$ for each channel between +14 and $+42 \mathrm{~km} \mathrm{~s}{ }^{-1}$ and after subtracting an appropriate background level for each channel. This mass, which is very approximate includes gas swept up by the SN shock and probably also ambient gas not yet reached by the blast wave. A rough estimation of the mass swept up by $3 \mathrm{C} 400.2$ can be derived by assuming that a $9.4 \mathrm{pc}$ diameter shell (the "small NW shell") expanded spherically into a uniformly distributed $21 \mathrm{~cm}^{-3}$ density ambient gas. In this way, the "small shell" swept up about $220 M_{\odot}$ of neutral hydrogen. Concerning the "large shell", it apparently expanded to the SE into a $4 \mathrm{~cm}^{-3}$ density medium and partially to the NW into the $21 \mathrm{~cm}^{-3}$ medium. Under these assumptions the "large shell" swept up roughly $540 M_{\odot}$.

The ambient density discontinuity which this remnant encounters will certainly affect the expansion velocity: the large shell is expected to attain larger velocities than the smaller one. This situation is in fact observed. We have shown in Fig. 4 the presence of $\mathrm{HI}$ features related to different portions of the "large shell" all along the velocity range, from +14 to $+42 \mathrm{~km} \mathrm{~s}^{-1}$; whereas for the "small NW shell", we detected associated features only between +16 and $+32 \mathrm{~km} \mathrm{~s}^{-1}$. From these evidences, we adopt as the expansion velocity for the "large shell" portion of the remnant, $14 \mathrm{~km} \mathrm{~s}^{-1}$, while for the "small shell", $8 \mathrm{~km} \mathrm{~s}^{-1}$. From the calculated masses and these partial expansion velocities, the kinetic energy transferred by 3C 400.2 to the surrounding neutral gas turns out to be about 1.2 $10^{48}$ ergs. This is a small portion of the probable initial energy of the explosion; however, we have to bear in mind that a good deal of energy is being presently radiated in form of optical filaments and in thermal X-ray. Also the derived masses and velocities are lower limits because neither molecular nor ionized gas contribution were included, and the determination of the extreme velocities can be seriously limited by galactic gas confusion.

If, on the other hand, we consider that the high velocity HI clouds seen projected onto the center of the remnant between -49.5 and $-44.5 \mathrm{~km} \mathrm{~s}^{-1}$ and probably the faint feature shown at $+69 \mathrm{~km} \mathrm{~s}^{-1}$, have been accelerated by the expanding shock front, then the expansion velocity turns out to be of the order of $70 \mathrm{~km} \mathrm{~s}^{-1}$. In this case the kinetic energy would be roughly $410^{49} \mathrm{ergs}$.

Tenorio-Tagle et al. (1985) investigated the dynamical evolution of SN explosions occurring near or inside molecular clouds. They predict that the morphology of the SNR depend upon the distance of the explosion site 
from the cloud boundary. The model which better fits the morphology observed in 3C 400.2 corresponds to a SN exploding close to the border of the cloud. In this case, two hemispherical remnants with different radii are produced: the smaller one within the cloud and a spherical large shell outside the cloud. Tenorio-Tagle et al. (1985) predict that for a breakout SNR in the Sedov phase the velocity ratio is related to the density ratio through the relationship $v_{\text {out }} / v_{\text {in }}=4.22-1.89\left(\log \left(n_{\text {out }} / n_{\text {in }}\right)+2\right)$, where out refers to the portion of SN expanding outwards from the cloud, and in points to the shock propagating inwards into the cloud. In the case of $3 \mathrm{C} 400.2$, there are regions that may have already become radiative as shown by the presence of optical filaments. We can however assume that the SNR is globally still in the adiabatic phase with cooling shock waves in denser clumps producing the optical emission. In this case, for a density contrast $n_{\text {in }} / n_{\text {out }} \simeq 5.2$, the velocity of the shock propagating into the cloud will be smaller by a factor of 1.8 , than that of the shock expanding outwards into the more diffuse ambient. This is in very good agreement with the observed velocity contrast $v_{\text {out }} / v_{\text {in }} \simeq$ 1.75 .

It has been suggested that a SNR expanding into a cloudy ISM is a plausible mechanism to produce centrally condensed X-ray morphology as observed in 3C 400.2 (White \& Long 1991). Our HI observations have revealed that the ambient interstellar medium where the remnant evolves has indeed a clumpy structure; but the observed contrast in densities between the cloud and intercloud media is much smaller than that assumed in the theoretic calculations. On the other hand, in order to explain the observed X-ray luminosity in 3C 400.2, Long et al. (1991) found that the intercloud density should be $0.04 \mathrm{~cm}^{-3}$. Our HI observations have demonstrated that the density of atomic gas in the vicinity of the SNR is at least two orders of magnitude higher, and the model fitting to the X-ray observations should be revised. An alternative scenario to produce similar morphology in the X-ray band was developed by Shelton (See review by Jones et al. 1998). This model assumes saturated thermal conduction as a mechanism to transport energy outwards from the very hot center. This may well be the mechanism at work in $3 \mathrm{C} 400.2$.

\section{Summary}

We performed HI $21 \mathrm{~cm}$ line observations in a $2.1 \times 2.1$ field around the SNR 3C 400.2 with the DRAO Synthesis Telescope. These observations revealed the existence of neutral gas features associated with the remnant. Namely: i) a clumpy $\mathrm{HI}$ cloud delineating the north, west and southern border of $3 \mathrm{C} 400.2$ in the velocity range +14 to $+42 \mathrm{~km} \mathrm{~s}^{-1}$ and ii) gas probably accelerated by the shock front at $v=-49.5$ and $+69 \mathrm{~km} \mathrm{~s}^{-1}$.

A simple picture that can explain the observed morphology of 3C 400.2 in the different spectral regimes is as follows: the remnant originated from a supernova explosion occurring near the border of a neutral gas cloud approximately $21 \mathrm{~cm}^{-3}$ in density. The position of the explosion coincides with the center of the HI void detected at $v=+27 \mathrm{~km} \mathrm{~s}^{-1}$ and with the geometrical center of the bright optical filaments. The "small NW shell" evolved within the dense cloud until it broke out originating the "large radio shell" where it is evolving into a medium about five times lighter. We argue that the optical filaments originated in regions where the front shock encountered and overtook density enhancements in the surrounding interstellar medium. The excellent correspondence between regions of $\mathrm{H} \alpha$ emission with regions of enhanced HI emission detected between +26 and $+28 \mathrm{~km} \mathrm{~s}^{-1}$ confirms this scenario. By comparing the HI observations with the X-ray image, we found a hole in the HI distribution at $+28 \mathrm{~km} \mathrm{~s}^{-1}$ coinciding exactly with the maximum in the X-ray emission, this suggests that the neutral gas could have been partially evaporated thus producing centrally condensed X-ray morphology.

From the present observations a distance of $2.8 \pm$ $0.8 \mathrm{kpc}$ to $3 \mathrm{C} 400.2$ was calculated.

Acknowledgements. We would like to thank Dr. Tom Landecker for his valuable contribution in the first stages of this work. E.B.G. thanks DRAO for support and the kind hospitality during her visit to Penticton. The Dominion Radio Astrophysical Observatory Synthesis Telescope is operated as a national facility by the National Research Council of Canada. This research was supported by the CONICET grant PMTPICT 0107.

\section{References}

Burton W.B., 1992, in The Galactic Interstellar Medium, D. Pfeniger \& P. Bartholdi (eds.). Springer-Verlag, p. 1

Caswell J.L., Lerche I., 1979, MNRAS 187, 201

Clark D.H., Caswell J.L., 1976, MNRAS 174, 267

Dubner G.M., Giacani E.B., Goss W.M., Winkler P.F., 1994, AJ 108, 207

Fich M., Blitz L., Stark A.A., 1989, ApJ 342, 272

Georgelin Y.M., Georgelin Y.P., 1976, A\&A 49, 57

Jones T.W., Rudnick L., Jun B.-I., et al., 1998, PASP (in press)

Long K.S., Blair W.P., White R.L., Matsui Y., 1991, ApJ 373, 567

Milne D.K., 1979, AuJPh 32, 83

Roger R.S., Costain C.H., Lacey J.D., Landecker T.L., Bowers F., 1973, Proc. IEEE 61, 1270

Rosado M., 1983, RMxA 8, 59

Saken J.M., Long K.S., Blair W.P., Winkler P.F., 1995, ApJ 443,231

Seward F.D., 1990, ApJS 73, 781

Tenorio-Tagle G., Bodenheimer P., Yorke H.W., 1985, A\&A 145,70

Veidt B.G., Landecker T.L., Vaneldik J.F., Dewdney P.E., Routledge D., 1985, Radio Sci. 20, 1118

White R.L., Long K.S., 1991, ApJ 373, 543

Winkler P.F., Olinger T.M., Westerbeke S.A., 1993, ApJ 405, 608 